\title{
Manejo anestésico para la cesárea en una paciente con síndrome de Klippel-Trenaunay. Reporte de un caso
}

\author{
Anesthetic management for cesarean section in a patient \\ with Klippel-Trenaunay syndrome. Report of a case
}

Sixto Fidel González Pérez ${ }^{1} \mathrm{MD}$, Nancy Morales León ${ }^{1} \mathrm{MD}$, Ana Cristina Caraballoso Morales ${ }^{1} \mathrm{MD}$, Yoslandy Díaz Carmenate ${ }^{1}$ MD, Mayuleisy Perdigón Hernández¹ MD.

\begin{abstract}
Klippel-Trenaunay syndrome is a rare disease that includes the triad of malformation of vascular capillaries, varicose veins and/or venous malformation and hypertrophy of soft or bony tissues. Its presence during pregnancy poses an anesthetic challenge due to the high obstetric risk due to thromboembolic and/or hemorrhagic phenomena. We present the case of a pregnant woman with the diagnosis of Klippel-Trenaunay syndrome announced for elective cesarean section. Our objective is to expose the anesthetic management carried out and to propose a strategic guide for anesthesiologists.
\end{abstract}

\section{RESUMEN}

El síndrome de Klippel-Trenaunay es una rara enfermedad que comprende la tríada de malformación de capilares vasculares, venas varicosas y/o malformación venosa e hipertrofia de tejidos blandos u óseos. Su presencia durante la gestación supone un reto anestésico por el alto riesgo obstétrico debido a fenómenos tromboembólicos y/o hemorrágicos. Presentamos el caso de una embarazada con el diagnóstico de síndrome de Klippel-Trenaunay anunciada para cesárea electiva. Nuestro objetivo es exponer el manejo anestésico realizado y proponer una guía estratégica para los anestesiólogos.

\section{Key words:}

Anaesthesia, cesarean section, Klippel-Trenaunay syndrome

\section{Palabras clave:}

Anestesia, cesárea, síndrome de Klippel-Trenaunay

Servicio de Anestesiología y Reanimación, Hospital General Provincial "Camilo Cienfuegos", Sancti Spíritus, Cuba.

Fecha de recepción: 10 de octubre de 2018

Fecha de aceptación: 03 de marzo de 2019

\section{ORCID}

https://orcid.org/0000-0001-9092-2444

Correspondencia:

Dr. Sixto Fidel González Pérez

Email: sixto.fidel@gmail.com 


\section{Introducción}

$\mathrm{E}$ I síndrome de Klippel-Trenaunay (SKT) está caracterizado por la presencia de várices, hemangiomas cutáneos, hipertrofia de tejidos blandos y huesos que contribuyen a la asimetría. También pueden estar presentes escoliosis, hiperhidrosis y trastornos de la coagulación. Las malformaciones vasculares, a menudo, se observan en: útero, médula espinal, columna vertebral, cerebro, hígado, intestinos, vejiga, bazo, riñones, sistema arterial, venoso y linfático, entre otras localizaciones[1]-[4] (Figura 1).

Este síndrome se describió por primera vez en 1900 por los médicos franceses Klippel y Trenaunay. Es un trastorno raro, y aún más cuando se presenta durante la gestación. La incidencia no está completamente conocida y son pocos los reportes en la paciente obstétrica. Se convierte, por lo anterior, en una característica complicada del embarazo[5]-[7].

Las manifestaciones clínicas presentes pueden agravarse en la parturienta debido a los cambios anatómicos y fisiológicos que sufre durante todo el proceso de gestación. El manejo anestésico para pacientes con síndrome de Klippel-Trenaunay solamente se ha descrito en informes de casos limitados[3],[7]. El objetivo de esta descripción es presentar la conducción anestésica de una gestante portadora del síndrome de Klippel-Trenaunay, sometida a cesárea electiva bajo anestesia general balanceada.

\section{Caso clínico}

Para la publicación del presente caso, se contó primeramente con la firma del consentimiento informado de la paciente y la autorización del Hospital General Provincial "Camilo Cienfuegos" de Sancti Spíritus, en Cuba, Consejo Científico y Comité de Ética Médica.

Desde la recepción hospitalaria de la paciente, el grupo de atención a la paciente obstétrica grave convocó una valoración multidisciplinaria (obstetricia, anestesiología, angiología, hematología, cuidados intensivos y Cirugía) que diseñó la estrategia para su manejo integral.

Paciente con 27 años de edad, $51 \mathrm{~kg}, 1,63 \mathrm{~cm}$, índice de masa corporal 19,2 kg/m², estado físico II según la clasificación de la $\mathrm{ASA}$, riesgo quirúrgico regular, 36,1 semanas de gestación y diagnóstico de síndrome de Klippel-Trenaunay, que es anunciada para cesárea electiva.

Datos positivos al interrogatorio y examen físico:

- Historia de sangrado ligero por traumas menores.

- Migraña vascular.

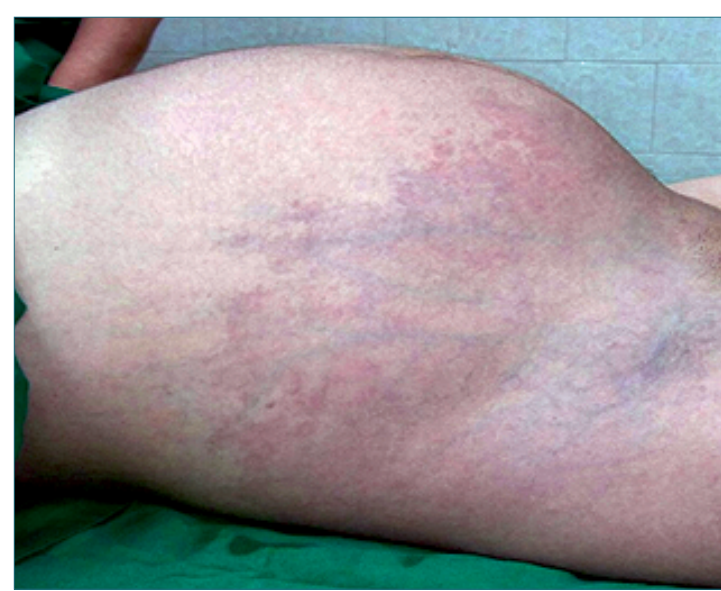

Figura 1. Extensión de las lesiones hemangiomatosas en el abdomen y hacia el lado derecho en la región torácica, lumbar y pélvica.

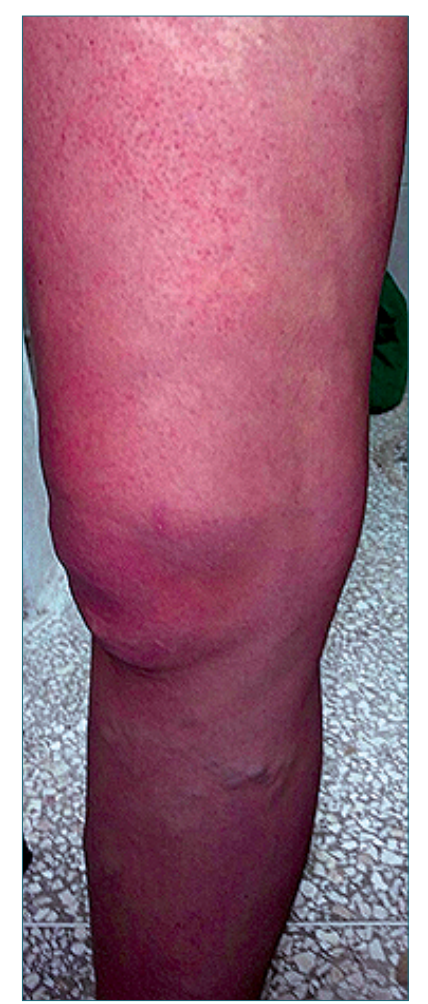

Figura 2. Miembro inferior derecho con lesiones hemangiomatosas y dilataciones venosas.

- Anemia ligera durante el embarazo.

- Hemangiomas cutáneos en el abdomen, miembro inferior derecho y hacia el lado derecho de la re- 
gión torácica, lumbar y pélvica.

- Dilataciones venosas en miembro inferior derecho (Figura 2).

- Asimetría de miembros inferiores.

- Signo de Mallampati grado 2.

Exámenes imagenológicos de interés:

- Tomografía axial computarizada (TAC) de abdomen con presencia de útero irregular, con múltiples várices en cara anterior y en región peritoneal parietal.

- Rayos X de columna vertebral con la observación de escoliosis por lesión ósea.

- Resonancia magnética nuclear (RMN) del neuroeje mostraba hemangiomas en el espacio espinal dorso-lumbar.

Exámenes de laboratorio:

- Hemoglobina 11,5 mg/dL, hematocrito 34\%, tiempo de sangramiento 1 minuto, tiempo de coagulación 8 minutos, conteo de plaquetas 290 x 10\%, INR 1,5, fibrinógeno $1,2 \mathrm{mg}$, tiempo de tromboplastina parcial activado 26 segundos, tiempo protombina 15 segundos, creatinina 0,8 $\mathrm{mg} / \mathrm{dL}$, glicemia 5,6 mmol/L, TGP 6 UI/L, TGO 10 UI/L y fosfatasa alcalina $57 \mathrm{UI} / \mathrm{L}$.

En la consulta preoperatoria, la paciente ofreció su consentimiento por escrito para el acto anestésico planificado. Se confirmó el empleo de medias de compresión venosa y la administración durante el embarazo de ácido fólico, fumarato ferroso y profilaxis antitrombótica $(0,3 \mathrm{ml}$ de fraxiheparina vía subcutánea cada 24 horas).

Al llegar a la sala preoperatoria, la gestante recibió la siguiente monitorización: oximetría de pulso $\left(\mathrm{SpO}_{2}\right)$, frecuencia respiratoria (FR), frecuencia cardiaca $(\mathrm{FC})$, presión arterial no invasiva (PANI) e invasiva (arteria radial), temperatura (axilar) mediante el DOCTUS VII. Se complementó con cardiotocografía externa, presión venosa central (PVC-vena yugular interna derecha) y diuresis. Se instaló una vía venosa periférica No 16 G recibiendo cefazolina $(2 \mathrm{~g})$, metoclopramida (10 mg), ranitidina (50 mg), solución Hartman $(500 \mathrm{ml})$ y cloruro de sodio $0,9 \%(500 \mathrm{ml}$ con $1 \mathrm{~g}$ de ácido tranexámico). Durante la estancia preoperatoria se colocó en posición lateral izquierda con administración de oxígeno vía catéter nasal (4 litros por minuto). Signos vitales iniciales: PANI 116/78 mmHg, FC 112 latidos por minuto, FR 16 respiraciones por minuto, $\mathrm{SpO}_{2} 100 \%$, temperatura $36,4^{\circ} \mathrm{C}$ y PVC $10 \mathrm{cmH}_{2} \mathrm{O}$.

En el período intraoperatorio, se continuó con la monitorización descrita y se incorporó la capnografía, temperatura (orofaringe), trazado electrocardiográfico y el control del bloqueo neuromuscular. Después de la preoxigenación, la inducción de la anestesia se obtuvo con fentanilo (50 mcg), lidocaína $(80$ mg), propofol $(100 \mathrm{mg})$ y succinilcolina $(50 \mathrm{mg})$ seguida de intubación endotraqueal en secuencia rápida con maniobra de Sellick, sin dificultades. Se acopló a la máquina de anestesia Mindray WATO EX-35 con parámetro ventilatorio en modo asistocontrolado por volumen. Para el mantenimiento, se utilizaron atracurio $(25 \mathrm{mg})$, fentanilo $(100 \mathrm{mcg})$, sevoflorano $(0,5$ CAM) con mezcla de $50 \%$ de oxígeno y $50 \%$ de aire. La cesárea se realizó con incisión paramedia y sección longitudinal uterina para extracción fetal. El recién nacido pesó 2.950 gramos, con Apgar de 8/9. Tras el nacimiento y la extracción placentaria, se administró oxitocina (20 U/I) en infusión continua para el control del sangrado. Pérdidas sanguíneas de $850 \mathrm{ml}$ y diuresis $400 \mathrm{ml}$. La administración hídrica se calculó a razón de $12 \mathrm{~mL} \cdot \mathrm{kg}^{-1} \cdot \mathrm{h}^{-1}$ con soluciones cristaloides. La paciente mantuvo estabilidad cardiorrespiratoria. El procedimiento quirúrgico tuvo una hora de duración. Previo a concluir, se administró ondansetrón (4 mg) vía intravenosa como profilaxis antiemética y morfina (5 mg por vía subcutánea) más diclofenaco (75 mg en infusión intravenosa continua) para el tratamiento del dolor postoperatorio. Al finalizar la intervención, se hizo reversión del bloqueo neuromuscular con posterior extubación.

La paciente fue llevada a la sala de recuperación posanestésica, consciente y orientada en ventilación espontánea con máscara de oxígeno y hemodinámicamente estable. Posteriormente, fue transferida a la Unidad de Cuidados Intensivos (UCI) para los cuidados posoperatorios donde su evolución ocurrió sin complicaciones.

\section{Discusión}

La presencia del síndrome de Klippel-Trenaunay durante el embarazo se clasifica como riesgo obstétrico alto debido a posibles complicaciones tromboembólicas y/o hemorrágicas, particularidad clínica que se asocia con múltiples comorbilidades relevantes y convierte este panorama en un reto para la anestesia obstétrica[3],[4],[8].

Los casos reportados son escasos y la incidencia de embarazo en pacientes portadoras del SKT no está clara, aproximadamente se reporta 1:27.500 nacimientos[8], por lo cual en la literatura científica la estrategia para la conducción anestésica no ha sido definida completamente[3],[7]. 
Los autores del presente artículo, teniendo en cuenta esta condición única, en este informe de caso, presentamos un análisis del manejo anestésico por acápites específicos, como se relaciona a continuación, con el objetivo de guiar a los anestesiólogos dedicados a la anestesia obstétrica:

- Pilares generales:

1. Diagnóstico precoz y diferencial.

2. Anamnesis y examen físico exhaustivos.

3. Exámenes evolutivos imagenológicos (radiografía simple, ecografía doppler, TAC, RMN, arteriografía, flebo y angiografía) y de laboratorio (énfasis en las pruebas de coagulación).

4. Identificación de comorbilidades.

- Regla de oro: Valoración integral multidisciplinaria [3],[4] (anestesiología, obstetricia, neonatología, radiología, angiología, urología, cirugía y medicina interna).

- Elección de la anestesia:

a. Consideraciones generales:

i. Consulta preoperatoria rigurosa (cumplir con los puntos 2, 3 y 4 de los pilares generales).

ii. Cumplir con las cinco reglas básicas de la práctica de la anestesia obstétrica[9].

iii. Medicación preanestésica para disminuir el sangrado intraoperatorio (ácido tranexámico) y evitar la aspiración broncopulmonar de contenido gástrico (metoclopramida, ranitidina y citrato de sodio $0,3 \mathrm{M})$.

iv. Evaluación del estado del binomio madre-feto.

v. Considerar la clasificación de la cesárea (electiva, urgente o emergente).

vi. Monitorización continua (cardiotocografía, $\mathrm{SpO}_{2}$, $F R$, capnografía, trazado electrocardiográfico, $F C^{\prime}$, PANI, presión arterial invasiva, temperatura, PVC, diuresis, ventilación mecánica, bloqueo neuromuscular e índice biespectral). La selección se hará de acuerdo con el estadio clínico del síndrome.

vii. Exámenes de laboratorio periódicos (periodo perioperatorio) para diagnóstico, evolución y tratamiento de trastornos de coagulación, tromboembólicos y hemorrágicos.

viii. Profilaxis antibiótica (cefazolina), antitrombótica (heparina de bajo peso molecular con bajas dosis de aspirina) y antiemética (biterapia).

ix. Estrategia multimodal para la analgesia posoperatoria (opioides y antiinflamatorios no esteroideos).

b. Anestesia general: La elección de esta técnica puede ser complicada para la intubación endotraqueal por las diversas condiciones clínicas de la enfermedad: hipertrofia de partes blandas, anomalías fáciles, anormalidades vasculares en el sistema venoso profundo, vía aérea con angiomas, edema y congestión vascular[3],[10]. Por lo anterior, se debe evitar o minimizar las repercusiones hemodinámicas derivadas de las maniobras de laringoscopia e intubación traqueal, con opioides y betabloqueantes[4]. La intubación endotraqueal puede ser más difícil; ante esta situación, se sugiere asegurar la vía aérea con técnicas de intubación despierta con fibra óptica y, si es significativo las malformaciones vasculares, considerar la máscara laríngea con presión continua del cricoides para minimizar el trauma de las vías respiratorias y evitar una hemorragia grave en las vías respiratorias[3].

c. Anestesia regional: Aunque en la gestante sometida a procedimientos obstétricos, la anestesia neuroaxial sea la más indicada con reconocida ventaja sobre la anestesia general, en este caso, se ve frustrada su selección por la presencia de fístulas arteriovenosas y hemangiomas en los espacios espinal y/o epidural que puede provocar su rotura por trauma con consecuente hemorragia, hematoma, compresión radicular y medular, que resulta en una lesión neurológica permanente[4],[11]. Algunos autores señalan que los bloqueos anestésicos regionales mayores pueden ser seguros cuando no haya evidencias inmediatas de anormalidades vasculares en la médula espinal y las pruebas evolutivas de coagulación sean normales[10],[11]. Recordar que la necesidad de profilaxis antitrombótica preoperatoria debe ser un punto importante a valorar, con la aplicación de la técnica regional según las normas internacionales en esta situación.

d. Recuperación posanestésica: Debe realizarse en una $\mathrm{UCl}$ por el alto riesgo obstétrico que representa.

La presencia de imágenes neuroaxiales determinó la elección de la anestesia general balanceada para la estrategia anestésica en esta paciente. Consideramos que la conducción realizada fue segura y efectiva; para ello, es indispensable que el anestesiólogo obtenga una comprensión completa del síndrome y su diagnóstico diferencial.

El éxito en el manejo anestésico del síndrome de Klippel-Trenaunay durante el embarazo requiere, en primer orden, de la participación de un equipo multidisciplinario con adecuada interrelación entre las especialidades involucradas, recordando que el acto anestésico será siempre individualizado. 


\section{Referencias}

1. Barajas Gómez TJ, Delgado Quiñones EG, Urióstegui Espíritu LC, López López V, Luna Breceda U. Síndrome de Klippel Trenaunay. Rev Cuba Med Gen Integral. 2016;32(3):1-7. Available from: http://scielo.sld.cu/pdf/mgi/ v32n3/mgi17316.pdf

2. Gaiser RR, Cheek TG, Gutsche BB. Major conduction anesthesia in a patient with KlippelTrenaunay Syndrome. J Clin Anesth. 1995 Jun;7(4):316-9. https://doi.org/10.1016/09528180(95)00046-K PMID:7546759

3. Sivaprakasam MJ, Dolak JA. Anesthetic and obstetric considerations in a parturient with Klippel-Trenaunay syndrome. Can J Anaesth. 2006 May;53(5):48791. https://doi.org/10.1007/ BF03022622 PMID:16636034

4. Teixeira CE, Braga AF, Braga FS, Carvalho VH, Costa RM, Brighenti GI. Anestesia para parto cesáreo em paciente portadora de síndrome de Klippel-Trenaunay. Rev Bras Anestesiol. $2018 \mathrm{Nov}$ - Dec;68(6):641-4. https://doi. org/10.1016/j.bjan.2018.01.015 PMID:29506860

5. Tanaka $R$, Fujita $Y$, Ishibashi Hiasa K, Yumoto Y, Hidaka N, Fukushima K, et al. Successful Management of Pregnancy Complicated by Klippel-Trenaunay Syndrome Using MR Angiography-Based Evaluation. Case Rep Obstet Gynecol. 2011;2011:723467. https:// doi.org/10.1155/2011/723467 PMID:22567516

6. Koch A, Aïssi G, Gaudineau A, Sananes N, Murtada R, Favre $\mathrm{R}$, et al. [Klippel-Trenaunay syndrome and pregnancy: difficult choice of delivery from a case and a review of the literature]. J Gynecol Obstet Biol Reprod (Paris). 2014 Sep;43(7):483-7. https://doi. org/10.1016/j.jgyn.2013.11.016 PMID:24461340

7. Dwivedi D, Sheshadri K, Tandon U, Chakraborty S. Klippel-Trenaunay syndrome: a rare entity with anesthesia concerns. J Clin Anesth. 2016 Dec;35:233-4. https://doi.org/10.1016/j.jclinane.2016.09.004 PMID:27871531

8. Chadha R. Management of Pregnancy with KlippelTrenaunay-Weber Syndrome: A Case Report and Review. Case Rep Obstet Gynecol. 2018 Jul;2018:6583562. https://doi. org/10.1155/2018/6583562 PMID:30112237

9. Wong Rosales MM. Reglas básicas para la práctica de la anestesia obstétrica. En: Canto Sánchez AL, Higgins Guerra LF, editores. Anestesia Obstétrica, 2 ed. México-Editorial El Manual Moderno;2008, p.185-89. https://www.casadellibro.com/ libro-anestesia-obstetrica2-ed/9789707293335/1251114

10. Barbara DW, Wilson JL. Anesthesia for surgery related to KlippelTrenaunay syndrome: a review of 136 anesthetics. Anesth Analg. 2011 Jul;113(1):98102. https://doi.org/10.1213/ ANE.0b013e31821a03c2 PMID:21467557

11. George SE, Sreevidya A, Asokan A, Mahadevan V. Klippel Trenaunay syndrome and the anaesthesiologist. Indian J Anaesth. 2014 Nov-Dec;58(6):775-7. https://doi. org/10.4103/0019-5049.147161 PMID:25624555 